\title{
Exploration of an Intelligent and Secure Wireless Body Area Networks for Health Monitoring
}

\author{
T.Santhi Vandana, S.Venkateshwarlu, CH.V.Ravi Teja
}

\begin{abstract}
Today's Wireless Body Area Networks technology augmented the wireless sensors communication more smart in the e-health line. With this sensors deployment prominent body parameters simply monitored remotely and the corresponding data is stored and transmitted the same for treatment purpose to the e-healthcare destination. Primarily Wireless Body Area Networks health care service is deliberately used in telemedicine, mobile health, elderly care, to monitor the chronic diseases and in emergency cases- the medical rehabilitations provided immediately without delay to the respective patient by the medical professional through internet service. As the demand of Wireless Body Area Networks e-health service increases, its ability is further enhanced once it is provided with more security and privacy to the enabled user's data with more integrity, confidentiality, availability features. Wireless Body Area Networks makes the people to improve their quality of life provided with affordable cost and flexibility in usage having minimum operation of events.

Researcher's shows an interest over the past years in e-health care services adaptability and concerns more on real-world complications. This survey paper mainly focuses on the conceptual structure of Wireless Body Area Networks communication, security and privacy issues, and allocated frequency bands.
\end{abstract}

Keywords - Wireless Body Area Networks, Security, Sensors, Frequency Bands, e-health.

\section{INTRODUCTION}

The Wireless Body Area Networks (WBAN's) is an interesting and widely accepted technology that created a special attention with it's friendly services in this smart world. Moreover as technology comes close to the people, the health perception with advanced e-health services makes friendly with the help of a great involvement of information technology. WBAN's e-health concept aims to enhance the functions and standards of the medical services, that in turn 's the quality of life improves with minimum hospitalization service cost.

Looking into WHO reports 17.7 million people and 80 percent of deaths are mainly due to heart attacks. The prime fact behind the Cardio Vascular Diseases (CVD's) are unbalanced diet, taking alcohol, lack of physical exercise's etc. All these harmful attacks of human beings can easily be controlled and reduced with healthy habits. Hopefully the death rates comes down with the help of WBAN's performance.[1] [Google Report's]

Revised Manuscript Received on July 10, 2019.

T.Santhi Vandana, Research Scholar, KLEF Deemed to be

University, Vaddeswaram, Guntur (Dt), A.P, INDIA (chary60@gmail.com)

S.Venkateshwarlu, Professor, KLEF Deemed to be University,

Vaddeswaram, Guntur (Dt), A.P, INDIA (somu23@kluniversity)

CH.V.Ravi Teja, Assistant Professor, Anurag College of Engineering,

Ghatkesar, Medchal (Dt), Telangana, INDIA. (chv.raviteja @gmail.com)
The biological body parameters like temperature, glucose level ,blood pressure, heart beat, brain activity, muscle functioning, digestive system and trunk position are monitored and measured by various wireless sensors namely temperature sensor, pulse sensor, electrocardiogram sensor, electroencephalogram, electromyography sensor and tilt sensor respectively and reports the same to the server through various wireless mediums.

With an interesting note that, the WBAN's sensors are a flavour of the Wireless Sensor Network's (WSN's) and these tiny wireless sensors are inserted in and around the patient's body or simply carrying the android/ios mobile phone (where all sensors are packed) which is provided with better coverage and superior channel capacity, less power utilization, acceptable radiation and other features made these to be well used in e-health care service. With all these sensors the structural health parameters can be easily monitored and tracked the corresponding data for the painless diagnosis, then the proper medicine at proper time can be prescribed in case of emergency demands, which could leads to improve the standard of life with technology aided. All these above tells us with the help of WSN's the user/patient can have great mobility experience and can be monitored whenever it is required. [1] [2][3]

These tiny wireless sensors not only used for patient's those are in hospital's or at remote location and also in numerous non- medical fields in order to enrich the quality of life, like in motion and gesture detection, for fitness monitoring during long time sitting, running, walking time, to provide guidance to the vehicle drivers, at the time of natural disaster, etc, the wireless sensors s are giving their best performance.[1] [2][3] [4]

Practically the WBAN's make up with three different devices with an intelligent binding, namely :

Sensor nodes : sometimes called as a mote, it is an electronic node in the sensor network, which is able to track the sensed information and communicate the same in the network line. This sensor node is build with power unit, processor cum memory unit, transceiver work and its hardware part.

Actuator: it is a component that performs its job upon the received sensor or control signal in the mechanical form. In e-health service it's role is to inject insulin, to restoring the activities, as per demand it pumps the heart for better functioning, or giving some sort of indications in the form of LED's and alarms. 


\section{EXPLORATION OF AN INTELLIGENT AND SECURE WIRELESS BODY AREA NETWORKS FOR HEALTH MONITORING}

Like as sensor nodes act uator consists of power unit, processor cum memory unit, transceiver unit and its hardware part.

Personal Digital Assistant(PDA) : this is technically called as handheld PC, which works as information managing device. Later replaced by smart phones with OS and android operating system. This unit also have its own power unit, processor cum memory unit, transceiver unit and its hardware part along with internet access that carries GPRS, Wi-MAX, GSM tracking .

The dynamic nature of the sensors creates the demand that all the people across the world are influenced to utilize for health updates for their prosperous of living standards .[5]

To deploy the authentic WBAN's service, still so many technical issues comes into picture in it's design structure.
The major issues that should concern in its design structure are security and privacy issues, its power source units, its size and wireless means of communication.

In this paper we mainly focus on the frame work of the WBAN's, security and privacy parameters and its solution as well as its frequency bands for better communication.

\section{FRAME WORK OF THE WIRELESS BODY AREA NETWORK'S}

As a matter of fact the WBAN's based e- health care services are basically user centric systems which give us real time health record and supervised by authorized persons with digitalized techniques. However this WBAN's is structured with three layers, shown in figure 1.

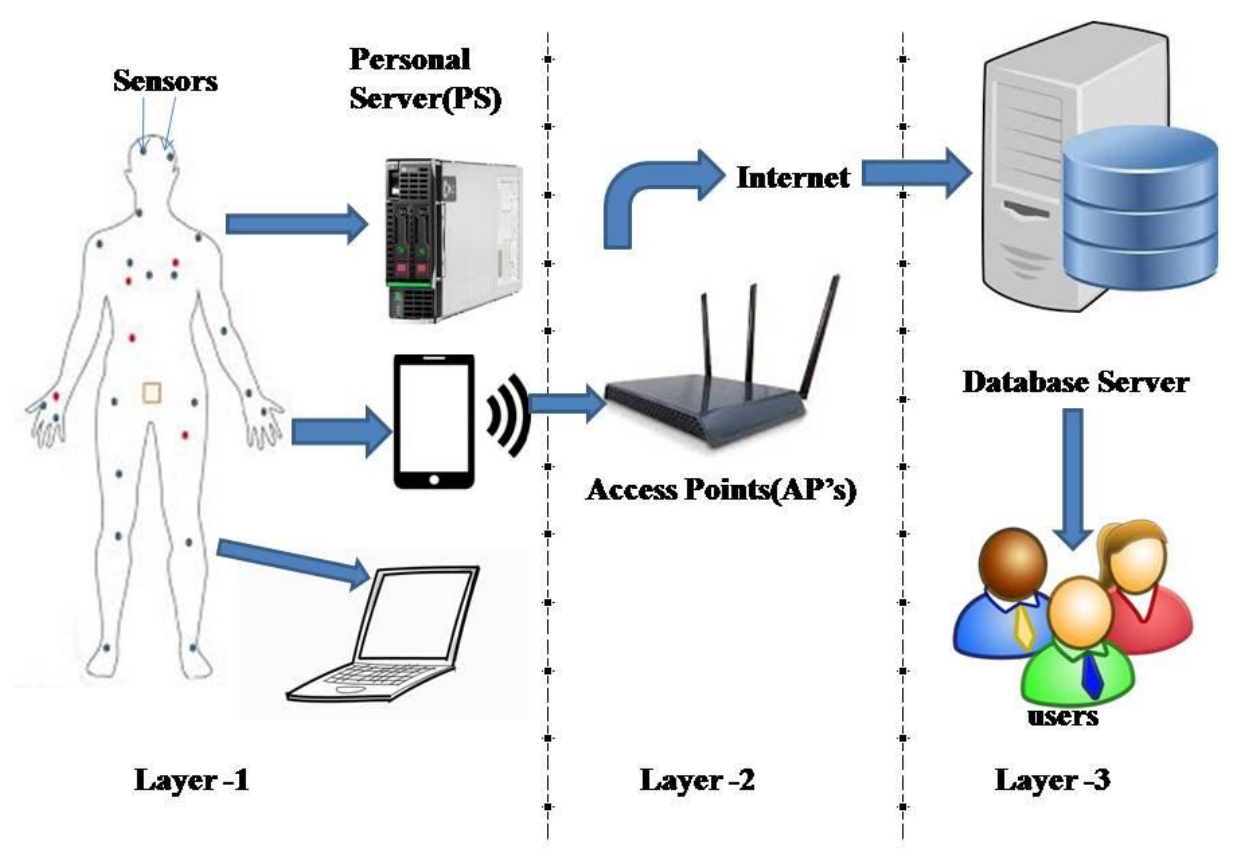

Figure 1:The frame work of Wireless Body Area Networks

Primarily the first layer is built with a group of smart biological sensors and Personal Server (PS). The main function of the personal server is to gather the sensed data from all the biological sensors those are implanted in and around the patient's body and behaves like a entree to the next level.

Subsequently the second layer establishes a connection between personal server (of the first stage) and Access Point's (AP's) which are located at various confined areas, provided with accessing permission and this layer is very helpful in emergency cases. This level of communication connects all the networks which connects the sensor and it can be accessed globally with the help of

Last the third layer is build with medical database server usually operates remotely since its stores patients information and health organization with their respective medical professor details, which helps to check the status of the patient's health record as per reports. [6] [7] internet service.

\section{ALLOTED FREQUENCY BANDS}

According to IEEE 802.15.6 Standard's the Wireless Body Area Networks (WBAN's) are operated well in different allocated frequency bands. Wireless biological sensors are low power operated devices and it requires certain frequency bands for the communication of sensor nodes, which are implanted in and around of the patients body or a common user who was having certain apps in the mobile phone. In WBAN's system the distinct frequency bands designated as HSB band, MICS band, WMTS band, ISM band, WMTS band, UWB band and the particular bands are abbreviated in the following table 1. The operating frequency starts from $5 \mathrm{Mhz}$ and ends with $10.6 \mathrm{Ghz}$ in which $2.4 \mathrm{Ghz}-2.48 \mathrm{Ghz}$ is widely accepted frequency band. [8] [9][10][11]

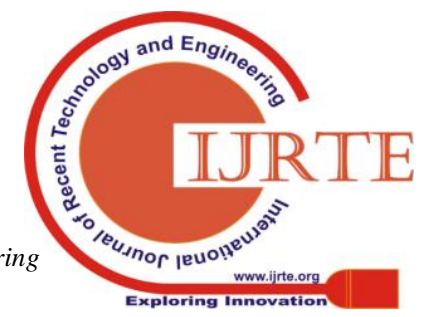


As per regulations of communications the first frequency band named as Human Body Communication (HBC) intend to transfer health information, human body tissues are itself acts like a transmitting media accounting with low power consumption, including with good signal propagation channels. The Medical Implant Communication Service (MICS) band is specified with 401- 406Mhz (core band of $402-405 \mathrm{Mhz}$ ), it is attributed with low power, means to operate at moderate range $(2 \mathrm{~m})$, delivering with a data rate of $400 \mathrm{Kbps}$, myriad of attributes used to design various implantable devices into the body like pacemakers, implanted cardiac defibrillators (ICD), as neuro stimulators to inject drugs along with baclofen pumps including with real time monitoring the heart function externally. As per Federal Communications Commission (FCC) the Wireless Medical Telemetry Service(WMTS) band subjected to wireless health services with a $14 \mathrm{Mhz}$ frequency, whereas operated with various frequency bands in different countries for medical application. Next the Industrial, Scientific and Medical band used world wide not only for medical diathermy systems, also supports for industrial, military, microwave ovens, and in mob ile phones provided with Bluetooth and Zigbee operated band (2.4 GHz -not requires any licence to use ). At last Ultra Wide Band range is well applicable to cancer tissues scanning with its imaging modality.

Table 1: Frequency bands of Wireless Body Area Networks

\begin{tabular}{|l|l|}
\hline Frequency Bands & Its Elucidations \\
\hline 5-50Mhz & $\begin{array}{l}\text { Human Body } \\
\text { Communication(HBC) }\end{array}$ \\
\hline 402-405Mhz & $\begin{array}{l}\text { Medical Implant Communication } \\
\text { Service Band(MICS )- } \\
\text { Implantable sensors [30] }\end{array}$ \\
\hline 420-450Mhz & $\begin{array}{l}\text { Wireless Medical Telemetry } \\
\text { Service(WMTS)- } \\
\text { Used in Japan }\end{array}$ \\
\hline 863-870Mhz & $\begin{array}{l}\text { Wireless Medical Telemetry } \\
\text { Service(WMTS)- } \\
\text { Used in Europe }\end{array}$ \\
\hline 902-928Mhz & $\begin{array}{l}\text { Industrial Scientific and } \\
\text { Medical(ISM)- } \\
\text { Basically used in Newzealand, } \\
\text { Australlia, North America[29] }\end{array}$ \\
\hline 2.4-2.48Ghz & $\begin{array}{l}\text { Industrial Scientific and } \\
\text { Medical(ISM)- } \\
\text { It is world wide used band but } \\
\text { suffered with interferences, as it not } \\
\text { requires any licence to use. }\end{array}$ \\
\hline $3.1-10.6 \mathrm{Ghz}$ & $\begin{array}{l}\text { UWB-supports for high data rates } \\
\text { with less interference[28] }\end{array}$ \\
\hline
\end{tabular}

\section{THE PREREQUISITES OF SECURITY AND PRIVACY ISSUES IN WBAN'S SYSTEM \& RESULTS}

\subsection{SECURITY AND PRIVACY REQUIREMENTS:}

To provide the means of WBAN's communication the security is a prerequisite parameter especially for health care applications. The most noticed threats that effects the performance of WBAN' system are (a) integrity (b) data confidentiality (c) privacy to access (d) data authentication (e) fault tolerence

Integrity: in general the quality of communication relies on how best the authorized users can access their information from the unique e-health database platform. To provide integrity one should know the rules to access their information from the database system to avoid malicious attacks, this means to notify that if all the people have permission to access this valuable and sensitive medical reports in public through internet, the morality of recorded data will be declined which seems harmful results effects the true patients in case if they need an immediate health service in emergency cases. To protect the data from unintentional modifications, proper authentication protocols need to create for true communication [7] [12]

Data confidentiality: it signifies that the WBAN's system must bound to absolute confidentiality to the patient's data, as it carries sensitive information. The various biological sensors detects the patients health condition and convey the same for the patients well-being to the e-health care system , so in this context provision of more protection from illegal users is an essential thing , otherwise it could leads danger to the patient life, some times it might experiences death to patient. So to encounter such dangerous happenings during transmission of the WBAN's information, the effective encryption techniques promises more confidentiality. Example : share key techniques provides secured transmission in between WBAN's nodes and its coordinators.[13][14][15][16][17][18][19][20]

Privacy to Access: it is immensely essential to provide more privacy to patient's health information. To accomplish this, the health organizations are need to adopt certain guide lines as per the government e-health regulations, moreover the same as to educate patients or users in order to access the data from the particular web site. For example the " American Health Insurance portability and Accountability Act (HIPPA)' and the 'Digital Information Security in Healthcare Act (DISHA)' comprised with a set of rules aims to give direction to the doctor's that they should maintain patient's e-health record up to certain years and protect the same, primarily not reveal it to public and at last such violation of the rules the government will take charge against the health organization's including professionals like civil and criminal offence act, so care must be taken. Including that patients should be aware to maintain their individual e-health record more safe and secured. [21] [22] [23] [24] [25] 


\section{EXPLORATION OF AN INTELLIGENT AND SECURE WIRELESS BODY AREA NETWORKS FOR HEALTH MONITORING}

Data Authentication: it signifies that to avoid malicious attacks from intruder, data authentication is one of the prerequisite parameter in medical as well as in nonmedical e-health applications. To put on value WBAN's system must validate that, the wireless communication taking place proper network and the coordinator nodes on an account of authentication. The Message between sensor nodes and networks to establish a trustworthy communication. [19][26] [27]

Fault Tolerance: one of the vital task of the WBAN'S system is to provide it's communication even if any fault appears.

\subsection{INTELLIGENCE OF SECURE WIRELESS BODY AREA NETWORKS:}

The smartness of Wireless Body Area Sensor restricted to disabled patient's, but also in modern days its gives fetching to all the people, to track the body parameters even in the absence of hospitals for the care of individuals -to take preventive measures or to approach the health professionals if at all any urgency required. The intelligence empowered in such a way that it can embed in shoes, belts and even in clothes like smart jacket monitoring ECG, also in terms of smart watches, etc.

\subsection{METHODS TO PROVIDE SECURITY TO WIRELESS BODY AREA NETWORKS:}

The flourish of the modern e-health service enchanting from the conventional health services to WBAN's service. To respect this, providing data security of the disabled individuals and the patients who need emergency hospitalization service is extremely essential. At present internet service is prerequisite, including with various networks for data management.

Cloud Computing: with the provision of internet service clients e-health data is being hosted by data service providers and the same can be retrieved with proper authentication and authorization by different cryptographic techniques based on computations with lightweight protocols to enhance the security.

True Random Binary Generator Technique: to provide more security and enrich confidence to the e-health users, the generation of random binary sequence generation to the real and live data is extensively used, now a days due to protect the data from hackers for a long time. Here are the true results shown in figures of an ECG signal that generates random signals continuously with 128 bits format with less computations to speed the communications from WBAN's to the destination following with different levels. Authentication Code (MAC) provides such validation Networks is enhancing day by day and its service is not

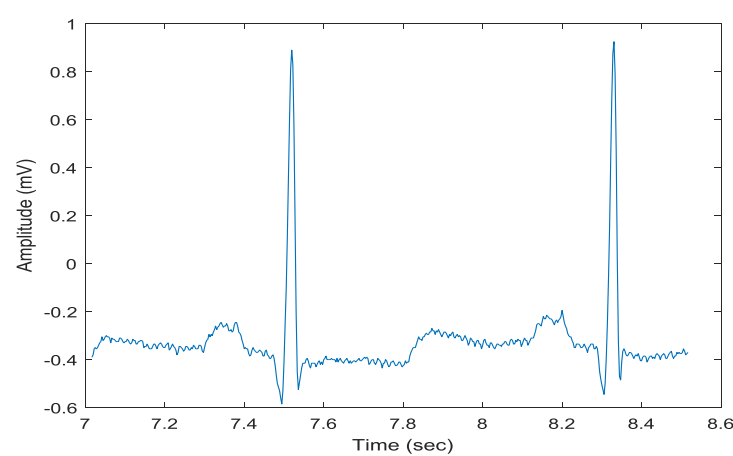

Figure 2. (a) Input ECG signal (b) RR interval measures using peak points

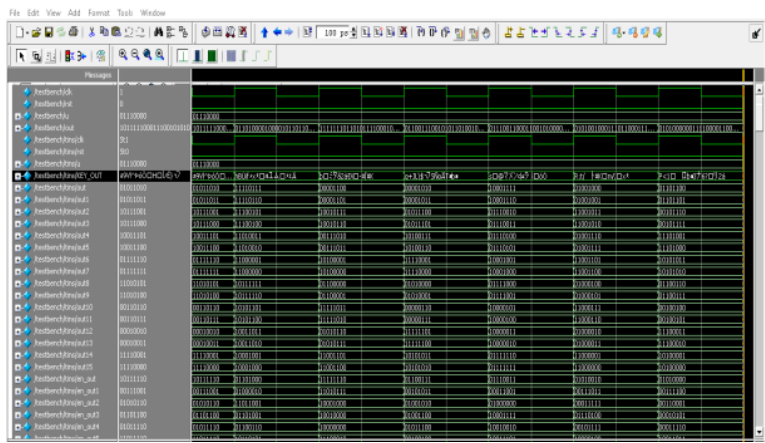

Figure 3. Randomized key sequence simulated output

\section{CONCLUSION:}

Clear to say that people are aware and effortlessly using the electronic health care systems with more concerns. In this context embedded sensors plays a key role in their day to day life activities to enrich the life standards further more. With the great involvement of information technology in Wireless Body Area Sensor Networks makes to promote the e-health services to the common people with an extreme level. This review work helped to present the structure of Wireless Body Area Networks (WBANs), it's various security and privacy issues, as well as to make a review on various frequency operating bands. As WBANs occupies the top-tier to impart e-health services among the people to sense, store and forward the same to health professionals for treatment purpose, well- being of the patients, the system structure should concern more on its security and privacy issues are exceptionally challenging task. To address the security concept more in communications the cryptographic techniques and random based generation methods vigorously used and some techniques will be specified here further elaborately described in future work.

\section{REFERENCES}

1. Amal Bouazizi, Ghada Zaibi, Mounir Samet, Abdennaceur Kachori, "Wireless Body Area Network for E-Health Applications:Overview". 978-1-5090-6323-9/17/\$31.00 2017 IEEE

2. Samaher Al-Janabi, Ibrahim Al-Shourbaji Mohammad Shojafar, Shahaboddin Shamshirband "Survey of Main

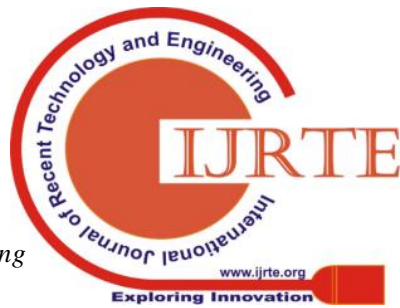


Challenges (Security and Privacy) in Wireless Body Area Networks for Healthcare Applications" Egyptian Informatics journal18(2017) 113-122.

3. Mohammad Ghamari, Balazs Janko, R.Simon Sherratt, William Harwin ,Robert Piechockoic, and Cinna Soltanpur "A Survey on Wireless Body Area Networks for eHealthcare Systems in Residential Environments" Sensors 2016,16,831; doi:10.3390/516060831.

4. Shasi Kant Shankar, Anurag Singh Tomar " A Survey on Wireless Body Area Network and Electronic -Healthcare" 978-1-5090-07774-5/16/\$31.00@ 2016 IEEE.

5. Rim Negra, Imen Jemili, Abdelfettah Belghith "Wireless Body Area Networks: Applications and Technologies" Procedia Computer Science 83(2016)1274-1281 1

6. Isra a Ahmed Zriqat ,Ahmad Mousa Altamini "Security and Privacy Issues in Ehealthcare Systems: Towards Trusted Services" International Journal of Advanced Computer Science and Applications(IJACSA) Vol.7, No. 9,2016

7. Patience E.Idoga, Mary Agoyi, Elizabeth Y.Coker-Farrell, Ogazi L.Ekeoma "Review of Security Issues in EHealthcare and Solutions" 978-1-5090-3784-1/16/\$31.00@ 2016 IEEE.

8. S.Kaur and Dr.J.Malhotra,"Performance Analysis of Channel model CM1 and CM2 1 of Wireless Body Area Networks". International Journal of Advanced Science and Technology, Vol.85,PP,43-54,2015.

9. J.Wang, Z.Zhang, Yuhui Zheng, Liwu Zuo and Jeong-UK Kim,"A Multitiers Service Architecture based Diabetes Monitoring for Elderly Care in Hospital". Internation Journal of Multimedia and Ubiquitous Engineering", Vol.8, No.3.PP 387-398, May 2013.

10. T.Yilmaz, T.Karacolak and E.Topskal," Characterization and Testing of a Skin Mimicing Material for Implantable Antennas Operating ISM Band (2.4GHz-2.48GHz)" IEEE Lett.Antennas and Wireless and Wireless Propag., Vol.7, PP.418-420,2018.

11. Y.J.Chi and F.C.Chen, "On-Body Adhesive-Bandage-Like Antenna for Wireless Medical Telemetry Service". In IEEE Transactions on Antennas and Propagation, Vol.62, No.5,PP 2472-2480, May 2014

12. Sapna Tyagi, Amit Agarwal, Piyush Maheshwari "A conceptual Framework for IOT- Based Healthcare System using Cloud Computing” 978-1-4673-8203-8/16/\$31.00@ 2016 IEEE.

13. Weh -Kuei Hsieh, Wen-Hsu Hsieh, Jiann- Liang Chen Chun-Yi Lin " Self-Configuration and Smart Binding Control on IOT Applications" ISBN 978-89-968650-6-3 Jan.31 Feb.3,2016 ICACT 2016.

14. Mohammad Kachuee, Mohammad Mandi Kiani, Hoda Mohammadzade, Mandi Shabanu "Cuff-Less Blood Pressure Estimation Algorithms for Continuous HealthCare Monitoring" 0018-9294(C)2016 IEEE

15. Carmen C, Peris-Loper P, Tapiador J.E(2015) “ Security and Privacy Issues in Implantable Medical Devices: A Comprehensive Survey" Journal of Biomedical Informatics 2015

16. Frontoni, E. Baldi,M., Zingaretti,P., Landro,V., and Misericordia, P(2014) October. "Security Issues for Data Sharing and Service Interoperability in E-health System: The Nu.Sa Test Bed". In Security Technology(ICCST), 2014 International Carnahan Conference on (PP 1-6). IEEE

17. Kargar MJ, Ghasemi S, Rahimi O.” Wireless Body Area Network: from Electronic Health Security Perspective". Int $J$ Reliable Quality E-healthcare (IJRQEH) 2013;2(4):38-47.

18. Ferdous MS, Chowdhury F, Moniruzzaman M. "A Taxonomy of Attack Methods on Peer-to-Peer Network", In: In the Proceedings of the First Indian Conference on Computational Intelligence and information Security. ICCIIS 2007.P 138-8
19. Han ND, Han L, Tuan DM, In HP, JoM.” A Scheme for Data Confidentiality in Cloud-Assisted Wireless Body Area Networks".Infsc, 2014; 284:157-66

20. Tewari A, Verma P.’Security and Privacy in E-health Monitoring with WBAN: A Critical Review. Int J Comput Appl 2016; 136(11)

21. Lim, Louw, Ren K."Data Security and Privacy in Wireless Body Area Networks".IEEE Wirel Commun 2010;17(1):51-8.

22. Al Ameen M, Liu J ,Kwak K.Security and Privacy Issues in Wireless Sensor Networks for Healthcare Applications". JMed Syst 2012;36(1):93-101.

23. Kumar R.Mukesh R."State of the Art: Security inWireless Body Area Networks" Int J Comput Sci Eng Technol(IJ CSET) 2013;4(5):622-30.

24. Somasundaram M.Sivakumar R.Security in Wireless Body Area Networks: A Survey. In: International Conference on Advancements in Information Technology ICBMG, IPCSIT, Singapore.p.20.

25. Office for Civil Rights ,United State Department of Health and Human Services. Medical Privacy National Standards of Protect the Privacy of Personal- Health Informationhttp://www.hhs.gov/ocr/Privacy/hipaa/ad ministrative/privacy rules index.html[accessed 20.12.15].

26. Digital Information Security in Heathcare Act(DISHA) https://www.nhp.gov.in

27. Kavitha T, Sridharan D."Security Valnerabilities in Wireless Sensor Networks: A Survey".J Inf Assurance Sec 2010:5(1):31-44.

28. Z.Mohammadi, R.Saadane, and Driss Aboutajdine," New High-Rate UWB Scheme for WBAN -Based Healthcare Systems", Progress in Electromagnetics Research B, Vol.60 PP.125-139, 2014

29. Jian Feng Zhao,Ximei Chen, Bo DongLiang and Qiu Xia Chen "A Review on Human Body Communication: Signal Propagation Model Communication Performance, and Experimental Issues". Wireless Communications and Mobile Computing

30. Mohd Noor Islam, Mehmet R.Yuce "Review of Medical Implant Communication System (MICS) Band and Network", http://www.science direct .com, 2016. 